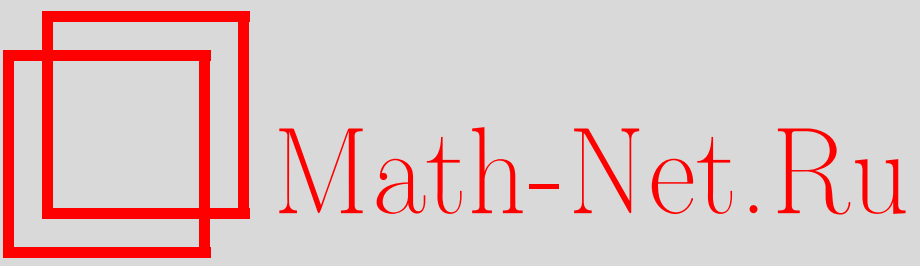

В. Д. Цуканов, Об определении статических напряжений деформированных солитонов, ТМФ, 2001, том 126, номер 2, 228-237

DOI: https://doi.org/10.4213/tmf427

Использование Общероссийского математического портала Math-Net.Ru подразумевает, что вы прочитали и согласны с пользовательским соглашением

http://www.mathnet.ru/rus/agreement

Параметры загрузки:

IP: 3.80 .181 .102

26 апреля 2023 г., 13:34:17 
ТЕОРЕТИЧЕСКАЯ

И МАТЕМАТИЧЕСКАЯ

ФИЗИКА

Том 126, № 2

февраль, 2001

(C) 2001 г.

В. Д. Цуканов*

\section{ОБ ОПРЕДЕЛЕНИИ СТАТИЧЕСКИХ НАПРЯЖЕНИЙ ДЕФОРМИРОВАННЫХ СОЛИТОНОВ}

На основе вариационного подхода к методу коллективных переменных дается ковариантный вывод уравнения для квазистатического солитонного анзаца, зависящего от произвольного набора коллективных переменных. Точно определяются полевая конфигурация и статические напряжения деформированного $\Phi^{4}$-кинка, обусловленные возбуждением внутренней солитонной моды. На примере нелинейной системы Клейна-Гордона рассмотрен вопрос об определении потенциала взаимодействия кинков на больших расстояниях. Обсуждается общий подход к проблеме точного определения межсолитонного потенциала для всей совокупности физически допустимых двухсолитонных конфигураций. Выясняется роль калибровочной инвариантности полученных уравнений в связи с неоднозначностью определения межсолитонного расстояния на той стадии сближения, при которой деформированные солитоны теряют свою индивидуальность.

\section{1. ВВЕДЕНИЕ}

При решении ряда солитонных задач вопросы динамики, по крайней мере на первом этапе, отходят на второй план и центральным моментом исследования становится определение статических напряжений в системе. Эта проблема непосредственно сводится к определению квазистатических солитонных конфигураций, описываемых с помошью набора неголдстоуновских коллективных координат. В этой связи можно указать на проблему определения межсолитонного потенциала в различных моделях. В этих задачах неголдстоуновскими коллективными координатами являются параметр разделения, а в многомерных случаях также и переменные, описьвающие относительную ориентацию солитонной пары во внутреннем пространстве. Кроме того, неголдстоуновские координаты, являющиеся, по сушеству, нелинейными амплитудами, описывают динамику внутренних солитонных мод. Проблема определения квазистатических конфигураций в этих задачах неоднократно обсуждалась, и пути ее решения связывались с различными модельными построениями. Здесь стоит отметить исследования взаимодействия скирмионов, которые были начаты еше в 1962 г. [1]-[7]. Кроме того, изучалось

\footnotetext{
${ }^{*}$ Институт теоретической физики, Национальный научный центр "Харьковский физико-технический институт", Харьков, Украина
} 
взаимодействие солитонов и в других моделях [8]-[10]. В этих работах были установлены важные свойства межсолитонных потенциалов. Вместе с тем очевидно, что подход, основанный на модельных, полуфеноменологических представлениях, не позволяет обеспечить всестороннего исследования проблемы. Подобное исследование может быть выполнено лишь в рамках последовательной теоретической схемы.

Касаясь этой проблемы, укажем на некоторые трудности, связанные с введением неголдстоуновских коллективных координат. Например, понятие расстояния между протяженными, испытываюшими взаимную деформацию объектами приобретает свой однозначный, наглядный смысл лишь в том случае, когда эти объекты достаточно далеко удалены друг от друга. Поэтому последовательная теория должна не только описывать процесс деформации солитонов по мере их сближения, но и содержать ответ на вопрос, как следует понимать неопределенность в определении расстояния между солитонами на той стадии сближения, когда в результате этой деформации солитоны теряют свою индивидуальность. Трудности построения теоретической схемы, приспособленной для описания межсолитонного взаимодействия, обусловленные этим обстоятельством, не раз отмечались в литературе и наиболее отчетливо были сформулированы в работе [11] Позднее в работе [12] были высказаны простые соображения в пользу универсального вариационного подхода к методу коллективных переменных, позволяюшего в рамках гамильтоновой процедуры формулировать различные задачи солитонной динамики. В этой же работе было получено общее уравнение для квазистатических конфигураций, определяемых произвольным набором коллективных координат. Сушественно то, что полученное уравнение инвариантно относительно репараметризации коллективных переменных, что обеспечивает независимость физических величин от способа определения межсолитонного расстояния.

В разделе 2 дается новый, ковариантный вывод уравнения для квазистатического анзаца, опирающийся на исходные понятия римановых многообразий. Следующие два раздела посвяшены разработке методов решения этого уравнения в конкретных случаях. В разделе 3 рассматривается вопрос об определении конфигурации и статических напряжений $\Phi^{4}$-кинка, деформация которого вызвана возбуждением внутренней солитонной моды. Данная задача решается точно. Ранее сходные вопросы на качественном уровне рассматривались во многих публикациях, ссылки на которые можно найти, например, в работах [13], [14]. На основе вариационного подхода динамика и релаксация внутренних мод рассматривались в работе [12]. Что касается проблемы определения межсолитонного взаимодействия, то вряд ли найдется модель с нетривиальным потенциалом, допускаюшая точное решение этой задачи. Если же интересоваться асимптотикой межсолитонного взаимодействия, то решение этой задачи, скорее всего, может быть найдено для любой модели. Пример такого расчета дается в разделе 4 , где уравнение для квазистатического анзаца используется для определения взаимодействия кинков нелинейного уравнения Клейна-Гордона в области больших межсолитонных расстояний. Следует подчеркнуть, что помимо самостоятельного интереса знание межсолитонного потенциала в асимптотической области позволяет ввести физически приемлемую калибровку при численном анализе уравнения во всем диапазоне межсолитон- 
ных расстояний. В разделе 5 обсуждаются результаты работы и отмечаются основные элементы построенной теоретической схемы.

\section{2. УРАВНЕНИЕ ДЛЯ КВАЗИСТАТИЧЕСКОГО АНЗАЦА}

Рассмотрим вопрос об определении квазистатического анзаца для солитонной системы, описываемой с помощью произвольного набора коллективных переменных. Пусть

$$
L=\sum g_{i k} \dot{q}^{i} \dot{q}^{k}-H(q, 0)
$$

- квадратичный по скорости невырожденный лагранжиан, допускаюший солитонные решения, $q^{i}$ - полевые переменные, $i \equiv\{i, x\}$ - совокупность дискретных индексов и пространственных координат. Исходное конфигурационное пространство удобно рассматривать как риманово многообразие с метрикой $g_{i k}$, порождаемой кинетическим членом. Тогда, если $Q$ - коллективные переменные, то квазистатический анзац $q_{c}^{i}(Q)$, описывающий деформированное солитонное состояние, можно интерпретировать как подмногообразие, параметризованное переменными $Q$. Будем считать, что этот анзац определяется из условия стационарности гамильтониана системы, заданного на $q_{c}^{i}(Q)$, относительно произвольных бесконечно малых вариаций $\delta q_{c}^{i}(Q)$, ортогональных поверхности этого подмногообразия, т.е.

$$
\frac{\partial H\left(q_{c}, 0\right)}{\partial q_{c}^{i}} \delta q_{c}^{i}=0
$$

где вариации $\delta q_{c}^{i}(Q)$ удовлетворяют условиям

$$
g_{i k}\left(q_{c}\right) \frac{\partial q_{c}^{i}(Q)}{\partial Q} \delta q_{c}^{k}(Q)=0
$$

Чтобы сократить вариации в уравнении (1), введем оператор проецирования $\mathcal{P}$ на касательное подпространство к подмногообразию $q_{c}^{i}(Q)$ в точке $Q$ :

$$
\mathcal{P}_{k}^{i}=g_{k l}\left(q_{c}\right) \frac{\partial q_{c}^{l}}{\partial Q} g^{Q Q} \frac{\partial q_{c}^{i}}{\partial Q}, \quad \mathcal{P}^{2}=\mathcal{P}
$$

где

$$
g_{Q Q}=\frac{\partial q_{c}^{i}}{\partial Q} g_{i k}\left(q_{c}\right) \frac{\partial q_{c}^{k}}{\partial Q}
$$

- метрика на подмногообразии $q_{c}^{i}(Q), g^{Q Q}$ - матрица, обратная к $g_{Q Q}$. Тогда, учитывая произвольность вариаций $\delta q_{c}^{i}(Q)$ в ортогональных к $q_{c}^{i}(Q)$ направлениях, уравнению (1) можно придать вид

$$
(1-\mathcal{P}) \frac{\partial H\left(q_{c}, 0\right)}{\partial q_{c}}=0
$$

откуда после подстановки выражения (2) для оператора $\mathcal{P}$ получим

$$
\frac{\partial H\left(q_{c}, 0\right)}{\partial q_{c}^{i}}=g_{i k}\left(q_{c}\right) \frac{\partial q_{c}^{k}}{\partial Q} g^{Q Q} \frac{\partial E(Q)}{\partial Q},
$$


где $E(Q) \equiv H\left(q_{c}(Q), 0\right)$ - энергия квазистатической конфигурации. В силу определения тензора $g^{Q Q}$ правая часть этого уравнения, учитываюшая наличие статических напряжений в коллективной подсистеме, инвариантна относительно произвольных невырожденных замен координат $Q \rightarrow Q^{\prime}(Q)$. Это указывает на ковариантный характер используемой вариационной процедуры. В этой связи стоит подчеркнуть, обрашаясь, например, к проблеме определения двухсолитонных конфигураций, что уравнение (3) точно описывает эти конфигурации в области расстояний, где деформированные солитоны теряют свою индивидуальность. Выбор же калибровки определяет лишш конкретный вид параметризации, описывающей эти конфигурации. Из соображений удобства можно потребовать, чтобы параметр разделения совпадал с естественным расстоянием между солитонами, когда это расстояние заметно превышает размер солитона. Заметим, что в случае, когда коллективные координаты являются голдстоуновскими переменными, т.е. когда координаты $Q$ совпадают с параметрами вырождения вакуумных решений, энергия $E$ не зависит от $Q$ и уравнение $(3)$ переходит в уравнение для статических решений $\partial H\left(q_{c}, 0\right) / \partial q_{c}^{i}=0$.

\section{3. СТАТИЧЕСКИЕ НАПРЯЖЕНИЯ ДЕФОРМИРОВАННОГО $\Phi^{4}$-КИНКА}

Воспользуемся уравнением (3) для определения конфигурации деформированного кинка в модели $\Phi^{4}$. Лагранжиан модели и соответствуюшее статическое кинковское решение $u_{c}(x-X)$, определенное с точностью до пространственной координаты $X$, имеют вид

$$
L=\frac{1}{2} \int d x\left(\dot{\Phi}^{2}(x)-\Phi^{\prime 2}(x)-\left(\Phi^{2}(x)-1\right)^{2}\right), \quad u_{c}(x)=\operatorname{th} x .
$$

Среди собственных функций оператора $\mathcal{L}$, описываюшего линейные флуктуации на фоне кинковского решения, имеется мода $\sigma(x)$, принадлежашая состояниям дискретного спектра:

$$
\mathcal{L} \sigma(x)=3 \sigma(x), \quad \sigma(x)=\sqrt{\frac{3}{2}} \frac{\operatorname{th} x}{\operatorname{ch} x}, \quad \mathcal{L}=-\frac{\partial^{2}}{\partial x^{2}}+4-6 \operatorname{ch}^{-2} x .
$$

При описании нестационарных состояний принято считать, что $\Phi^{4}$-кинк обладает внутренней степенью свободы, малые колебания которой можно отождествить с модой $\sigma(x)$. Поэтому набор коллективных координат, описываюших кинковское состояние, помимо трансляционной координаты $X$ должен включать также переменную $\tau$, связанную с возбуж дением внутренней моды $\Phi^{4}$-кинка. Можно ожидать, что при таком наборе коллективных координат образуюшие $\Phi_{c}^{\prime} \equiv \partial \Phi_{c} / \partial x, \Phi_{c \tau} \equiv \partial \Phi_{c} / \partial \tau$, построенные на решениях уравнения (3), будут ортогональны в касательном пространстве:

$$
\int d x \Phi_{c}^{\prime}(x) \Phi_{c \tau}(x) \equiv\left\langle\Phi_{c}^{\prime} \Phi_{c \tau}\right\rangle=0
$$

Учитывая также, что энергия не зависит от циклической переменной, уравнение (3) в рассматриваемом случае можно представить в виде

$$
-\Phi_{c}^{\prime \prime}(x)+2 \Phi_{c}(x)\left(\Phi_{c}^{2}(x)-1\right)=\Phi_{c \tau}(x)\left\langle\Phi_{c \tau}^{2}\right\rangle^{-1} \frac{\partial E}{\partial \tau} .
$$


Правая часть этого уравнения инвариантна относительно репараметризации $\tau \rightarrow \tau^{\prime}(\tau)$. Если зафиксировать коллективную переменную с помошью калибровки

$$
-\left\langle\Phi_{c \tau}^{2}\right\rangle^{-1} \frac{\partial E}{\partial \tau}=1
$$

то уравнение (7) переходит в нелинейное уравнение диффузии

$$
\Phi_{c \tau}(x)-\Phi_{c}^{\prime \prime}(x)+2 \Phi_{c}(x)\left(\Phi_{c}^{2}(x)-1\right)=0 .
$$

Это уравнение исследовалось в работе [15], где были найдены его точные решения. В частности, решения в виде деформированного кинка, стремящиеся к +1 при $x \rightarrow+\infty$, можно записать в виде

$$
\Phi_{c}(x, \tau)=\frac{e^{2 x}-1}{e^{2 x}+1 \pm e^{x-3 \tau}} .
$$

Выполняя интегрирование, легко убедиться, что эти решения удовлетворяют условию ортогональности (6) и калибровочному условию (8). Заметим теперь, что физически актуальная область мальх деформаций, где решения (10) лишь незначительно отличаются от статического кинковского решения (4), соответствует значениям $\tau \rightarrow \infty$. Другими словами, коллективная переменная $\tau$, определяемая калибровочным условием (8), неудобна с физической точки зрения и играет лишь промежуточную роль, позволяя представить уравнение (7) в виде нелинейного уравнения диффузии. Поэтому пару решений (10) целесообразно объединить в одно:

$$
\Phi_{c}(x, q)=\frac{e^{2 x}-1}{e^{2 x}+1-\sqrt{6} q e^{x}} \approx u_{c}(x)+q \sigma(x)+O\left(q^{2}\right),
$$

где новая коллективная переменная $q$, принимающая как положительные, так и отрицательные значения, при малых деформациях имеет смысл амплитуды внутренней моды [12]. Энергия конфигурации (11) $E(q) \equiv H\left(\Phi_{c}, 0\right)$, определяющая статические напряжения деформированного кинка, имеет вид

$$
E(q)=\frac{4}{3}+\frac{\bar{q}^{2}}{1-\bar{q}^{2}}-\frac{\bar{q}^{3}}{\left(1-\bar{q}^{2}\right)^{\frac{3}{2}}} \arccos \bar{q} \approx \frac{4}{3}+\frac{3}{2} q^{2}+O\left(q^{3}\right), \quad \bar{q}=\sqrt{\frac{3}{2}} q .
$$

Здесь первое слагаемое представляет собой массу кинка, а член, квадратичный по $q$, описывает потенциал гармонического осциллятора с частотой внутренней моды $\omega^{2}=3$. Область изменения коллективной координаты, в которой энергия остается вещественной, ограничена интервалом $-\sqrt{2 / 3}<q<+\sqrt{2 / 3}$. В этой области значений $q$ решение (11) не имеет особенностей по пространственной переменной. Подчеркнем также, что выбор физической коллективной координаты, стремяшейся в пределе малых деформаций к амплитуде внутренней моды, неоднозначен, определяется соображениями удобства и в рассматриваемом случае связан с явным видом решений для деформированного кинка (10). 


\section{4. О ВЗАИМОДЕЙСТВИИ КИНКОВ}

Важную роль в исследовании свойств солитонных состояний играют решения уравнения $(9)$, определяемые асимптотикой $\Phi_{c}(x) \rightarrow-1$ при $x \rightarrow \pm \infty$. Эти решения описывают конфигурации кинк-антикинк и позволяют установить свойства межсолитонного взаимодействия. Коллективная переменная $\tau$ в этом случае связана с параметром разделения $R$, а возбуждения внутренних мод не учитываются. K сожалению, точные решения уравнения (9) с данными граничными условиями, по-видимому, отсутствуют [16]. Приближенные же решения в области больших расстояний $R$ можно получить в общем виде, рассмотрев вместо (7) соответствующее уравнение для нелинейной модели Клейна-Гордона

$$
-\Phi_{c}^{\prime \prime}(x)+U^{\prime \prime}\left(\Phi_{c}(x)\right)=\Phi_{c R}(x)\left\langle\Phi_{c R}^{2}\right\rangle^{-1} \frac{\partial E}{\partial R},
$$

где $U(\Phi)$ - потенциал модели. Если поместить начало координат в центр бисолитонной пары и учесть ее симметрию $\Phi_{c}(x, R)= \pm \Phi_{c}(-x, R)$, то всю конфигурацию можно описать, рассмотрев область $x \leqslant 0$. Здесь четные функции описывают кинк-антикинковские состояния, нечетные - кинк-кинковские (в случае периодического потенциала $U(\Phi))$. Когда расстояние $R$ между солитонами велико, то слева от точки $x=0$ анзац $\Phi_{c}(x, R)$ мало отличается от однокинковского решения $u_{c}(x+R / 2)$ статического уравнения Клейна-Гордона

$$
-u_{c}^{\prime \prime}(x)+U^{\prime}\left(u_{c}(x)\right)=0 .
$$

Поэтому, представляя анзац $\Phi_{c}(x, R)$ в виде

$$
\Phi_{c}(x, R)=u_{c}\left(x+\frac{R}{2}\right)+\eta\left(x+\frac{R}{2}, R\right), \quad x \leqslant 0,
$$

где функция $\eta(x+R / 2, R)$ стремится к нулю при $R \rightarrow \infty$, и замечая, что в области больших $R$

$$
2\left\langle\Phi_{c}^{\prime 2}\right\rangle \approx \int_{-\infty}^{\infty} d x u_{c}^{\prime 2}(x) \equiv M,
$$

где $M$ - масса кинка, из (12) найдем уравнение для $\eta(x)$ :

$$
\mathcal{L} \eta(x)=-\frac{1}{M} \frac{\partial E}{\partial R} u_{c}^{\prime}(x), \quad x \leqslant \frac{R}{2}, \quad \mathcal{L} \equiv-\frac{\partial^{2}}{\partial x^{2}}+U^{\prime \prime}\left(u_{c}(x)\right),
$$

где $\partial E / \partial R$ - малый параметр в области больших $R$. Умножая уравнение (15) на нулевую моду $u_{c}^{\prime}(x)$ оператора $\mathcal{L}$ и интегрируя по частям в пределах от $-\infty$ до $x$, можно понизить порядок уравнения. В результате получим следующее линейное уравнение первого порядка для функции $\eta(x)$ :

$$
-u_{c}^{\prime}(x) \eta^{\prime}(x)+u_{c}^{\prime \prime}(x) \eta(x)=\frac{M(x)}{M} \frac{\partial E}{\partial R}, \quad M(x)=\int_{-\infty}^{x} d x^{\prime} u_{c}^{\prime 2}\left(x^{\prime}\right) .
$$

Решение этого уравнения можно представить в виде

$$
\eta(x)=u_{c}^{\prime}(x)\left\{\frac{\eta\left(\frac{R}{2}\right)}{u_{c}^{\prime}\left(\frac{R}{2}\right)}-\frac{1}{M} \frac{\partial E}{\partial R} \int_{x}^{\frac{R}{2}} d x^{\prime} \frac{M\left(x^{\prime}\right)}{u_{c}^{\prime 2}\left(x^{\prime}\right)}\right\} .
$$


Функция $\eta(R / 2)$, играющая в этой формуле роль константы интегрирования, определяется из граничных условий. Для пар кинк-антикинк и кинк-кинк эти условия имеют вид соответственно $\Phi_{c}^{\prime}(0, R)=0$ и $\Phi_{c}(0, R)=u_{+}$, где $u_{ \pm} \equiv u_{c}( \pm \infty)$ - вакуумные константы. Используя эти условия и уравнение (16), получим следующие выражения для граничных значений $\eta(R / 2), \eta^{\prime}(R / 2)$ в обоих этих случаях:

$$
\eta^{\prime}\left(\frac{R}{2}\right)=-u_{c}^{\prime}\left(\frac{R}{2}\right), \quad \eta\left(\frac{R}{2}\right)=\frac{1}{u_{c}^{\prime \prime}\left(\frac{R}{2}\right)}\left\{\frac{M\left(\frac{R}{2}\right)}{M} \frac{\partial E}{\partial R}-u_{c}^{\prime 2}\left(\frac{R}{2}\right)\right\}
$$

для пары кинк-антикинк и

$$
\begin{aligned}
\eta\left(\frac{R}{2}\right) & =u_{+}-u_{c}\left(\frac{R}{2}\right) \\
\eta^{\prime}\left(\frac{R}{2}\right) & =-\frac{1}{u_{c}^{\prime}\left(\frac{R}{2}\right)}\left\{\frac{M\left(\frac{R}{2}\right)}{M} \frac{\partial E}{\partial R}+u_{c}^{\prime \prime}\left(\frac{R}{2}\right)\left(u_{c}\left(\frac{R}{2}\right)-u_{+}\right)\right\}
\end{aligned}
$$

для пары кинк-кинк. Решение (17) описывает искажение кинка, вызванное влиянием второго солитона, когда расстояние между кинками сушественно превосходит их размеры и зависит от малого параметра $\partial E / \partial R$, определяюшего взаимодействие солитонов. Заметим, что формулы (17)-(19) включают члены более высокого порядка по сравнению с членами главной асимптотики по $R$. Однако селекция этих членов при рассмотрении полевой переменной $\eta(x)$ нецелесообразна, поскольку функция $\eta(x)$ содержит конкурирующие пространственные экспоненты. Поэтому формулы (17)-(19) следует использовать для корректного определения в главном приближении интегральной величины межкинковского потенциала $E(R)$. Так как $(1 / 2) u_{c}^{\prime 2}=U\left(u_{c}\right)$, то после подстановки выражения (13) в формулу для энергии $E(R)=H\left(\Phi_{c}, 0\right)$, разложения по $\eta(x)$ и интегрирования по частям с учетом уравнения (16) получим

$$
\begin{aligned}
E(R)-2 M= & -2 \int_{\frac{R}{2}}^{\infty} d x u_{c}^{\prime 2}(x)+\frac{1}{M} \frac{\partial E}{\partial R} \int_{-\infty}^{\frac{R}{2}} d x \eta(x) u_{c}^{\prime}(x)+ \\
& +\eta\left(\frac{R}{2}\right)\left(2 u_{c}^{\prime}\left(\frac{R}{2}\right)+\eta^{\prime}\left(\frac{R}{2}\right)\right)
\end{aligned}
$$

Подставляя в (20) выражение для $\eta(x)(17)$ и используя граничные значения (18) и (19) для пар кинк-антикинк и кинк-кинк, убеждаемся, что линейные по $\partial E / \partial R$ члены исчезают. При этом соотношение (20) принимает вид

$$
E(R)-2 M=A(R)+B(R)\left(\frac{1}{M} \frac{\partial E}{\partial R}\right)^{2}
$$

где функции $A(R), B(R)$ определяются формулами

$$
\begin{aligned}
& A(R)=-\frac{u_{c}^{\prime 3}\left(\frac{R}{2}\right)}{u_{c}^{\prime \prime}\left(\frac{R}{2}\right)}-2 \int_{\frac{R}{2}}^{\infty} d x u_{c}^{\prime 2}(x) \\
& B(R)=\int_{-\infty}^{\frac{R}{2}} d x u_{c}^{\prime 2}(x)\left\{\frac{M\left(\frac{R}{2}\right)}{u_{c}^{\prime}\left(\frac{R}{2}\right) u_{c}^{\prime \prime}\left(\frac{R}{2}\right)}+\int_{x}^{\frac{R}{2}} d x^{\prime} \frac{M\left(x^{\prime}\right)}{u_{c}^{\prime 2}\left(x^{\prime}\right)}\right\}
\end{aligned}
$$


для пары кинк-антикинк и

$$
\begin{aligned}
& A(R)=\left(u_{+}-u_{c}\left(\frac{R}{2}\right)\right)\left\{2 u_{c}^{\prime}\left(\frac{R}{2}\right)+\frac{u_{c}^{\prime \prime}\left(\frac{R}{2}\right)}{u_{c}^{\prime}\left(\frac{R}{2}\right)}\left(u_{+}-u_{c}\left(\frac{R}{2}\right)\right)\right\}-2 \int_{\frac{R}{2}}^{\infty} d x u_{c}^{\prime 2}(x), \\
& B(R)=\int_{-\infty}^{\frac{R}{2}} d x u_{c}^{\prime 2}(x) \int_{x}^{\frac{R}{2}} d x^{\prime} \frac{M\left(x^{\prime}\right)}{u_{c}^{\prime 2}\left(x^{\prime}\right)}
\end{aligned}
$$

для пары кинк-кинк. Асимптотика кинковского решения имеет вид

$$
\left.u_{c}(x)\right|_{x \rightarrow \pm \infty} \rightarrow u_{ \pm} \mp a e^{\mp m x}
$$

где $m=\sqrt{U^{\prime \prime}\left(u_{ \pm}\right)}$- масса пиона. Учитывая, что $M(\infty)=M, M(-\infty)=0$, и вычисляя коэффициенты $A(R), B(R)$ в главном приближении по $e^{-m R}$, убеждаемся, что функции $A(R)$ исчезают, а $B(R)$ совпадают с точностью до знака. Поэтому в главном приближении уравнение (21) принимает вид

$$
E(R)-2 M=\mp \frac{1}{2 m^{3} a^{2}} e^{m R}\left(\frac{\partial E}{\partial R}\right)^{2} .
$$

Отсюда находим энергию взаимодействия бисолитонной пары на больших расстояниях

$$
E(R)=2 M \mp 2 m a^{2} e^{-m R} .
$$

Эта формула описывает притяжение и отталкивание для конфигураций кинк-антикинк и кинк-кинк, соответственно. Применительно к системам $\Phi^{4}$ и синус-Гордон выражение для энергии (22) было получено в работе [8] с помошью приближенного уравнения типа (12), в котором правая часть имитировалась $\delta$-образным феноменологическим источником [17]. Для определения конфигурации солитонов и их взаимодействия во всем диапазоне расстояний $R$ следует зафиксировать калибровку и численно решить уравнение (12). Заметим, что представление решения в виде (13) частично фиксирует коллективную переменную в области больших межсолитонных расстояний. Полученные на основе разложения (13) формулы (14) и (22) могут быть использованы для введения естественной калибровки

$$
\left\langle\Phi_{c R}^{2}\right\rangle^{-1} \frac{\partial E}{\partial R}= \pm \frac{(2 m a)^{2}}{M} e^{-m R}
$$

обеспечивающей совпадение параметра $R$ с истинным расстоянием между солитонами в асимптотической области $R \rightarrow \infty$. После фиксации калибровки (23) уравнение (12) принимает вид нелинейного уравнения диффузии

$$
-\Phi_{c}^{\prime \prime}(x)+U^{\prime \prime}\left(\Phi_{c}(x)\right)= \pm \frac{(2 m a)^{2}}{M} e^{-m R} \frac{\partial \Phi_{c}(x)}{\partial R} .
$$


С помощью этого уравнения можно определить поправки к потенциалу (22) в асимптотической области в данной калибровке, а его численное решение позволяет воспроизвести всю совокупность физически допустимых двухсолитонных конфигураций. Подчеркнем, что решение этого уравнения в случае конфигураций кинк-антикинк соответствует описанию диффузии в положительном направлении "времени" $-R$. Такой процесс является устойчивым, и сближение пары кинк-антикинк завершается ее "аннигиляцией", при которой функция $\Phi_{c}(x)$ переходит в вакуумную константу [16]. Фактически расчет эволюции пары кинк-антикинк, выполненньй в работе [16] в связи с исследованием нелинейного уравнения диффузии, является примером строгого определения всей совокупности двухсолитонных конфигураций в задачах, связанных с изучением межсолитонного взаимодействия на основе уравнения (3). Что касается построения конфигураций кинк-кинк, то эта задача сводится к изучению диффузии в отрицательном направлении “времени” $R$. В области больших межсолитонных расстояний наличие нелинейности в уравнении (24) стабилизирует неустойчивость такой процедуры, характерную для линейной теории. По мере сближения стабилизация уменьшается и предельная полевая конфигурация определяется точкой, в которой устойчивость такого процесса обрывается.

\section{5. ЗАКЛЮЧЕНИЕ}

В работе на основе вариационного подхода дан ковариантный вьвод уравнения для квазистатического анзаца. Характерной особенностью подхода является наличие репараметризационной инвариантности по отношению к замене коллективных переменных. Циклические переменные либо переменные, связанные с вырождением вакуумных решений, вводятся стандартным образом. Произвол же в выборе переменных неголдстоуновской природы устраняется из физических соображений в области, где этим переменным можно придать непосредственный физический смысл. В остальном их выбор определяется соображениями удобства. В качестве примера рассмотрены статические деформации $\Phi^{4}$-кинка, связанные с возбуждением внутренней моды. Данная задача решается точно. Определены конфигурация деформированного кинка, его энергия и физически допустимая область изменения амплитуды нелинейных колебаний. Затронут также вопрос об определении межсолитонного потенциала для нелинейного уравнения Клейна-Гордона. В области больших расстояний уравнение для квазистатического анзаца линеаризуется, что позволяет установить деформацию кинков и асимптотический характер взаимодействия. Последнее обстоятельство позволяет ввести естественную калибровку и представить уравнение для квазистатического анзаца в виде нелинейного уравнения диффузии, приспособленного для численного определения всей совокупности физически допустимых дву хсолитонных конфигураций. Говоря о проблеме определения межсолитонного потенциала, следует подчеркнуть, что хотя для большинства актуальных моделей двухсолитонные конфигурации подверглись всестороннему изучению с помощью различных качественных подходов, исследования на основе уравнения (3) позволяют точно учесть деформацию солитонов на малых расстояниях, т.е. в области, наиболее чувствительной к дефектам, свойственным искусственным полевым конст- 
рукциям. Это особенно важно, например, в модели Скирма, так как в этой области нуклон-нуклонный потенциал имеет незначительный минимум, формируюший энергию связи ядер. Хотя уравнение (3) не учитывает спиновых эффектов, изучение на его основе скирмион-скирмионного взаимодействия является необходимым этапом в процедуре определения нуклон-нуклонного потенциала.

Благодарности. Автор благодарен П. О. Мчедлову-Петросяну и В. В. Ганну за ряд полезных обсуждений.

\section{Список литературы}

[1] T. H. R. Skyrme. Nucl. Phys. B. 1962. V. 31. P. 556.

[2] A. Jackson, A. D. Jackson, V. Pasquier. Nucl. Phys. A. 1985. V. 432. P. 567.

[3] E. Sorace, M. Tarlini. Phys. Rev. D. 1986. V. 33. P. 253.

[4] В. Б. Копелиович, Б. Е. Штерн. Письма в ЖЭТФ. 1987. Т. 45. С. 165.

[5] T.S. Walhout, J. Wambach. Phys. Rev. Lett. 1991. V. 67. P. 314.

[6] G. Kalbermann. Nucl. Phys. A. 1993. V. 561. P. 682.

[7] N.R. Walet, R. D. Amado. Phys. Rev. C. 1993. V. 47. P. 498.

[8] R. Rajaraman. Phys. Rev. D. 1977. V. 15. P. 2866.

[9] N.S. Manton. Nucl. Phys. B. 1977. V. 126. P. 525.

[10] L. Jacobs, C. Rebbi. Phys. Rev. B. 1979. V. 19. P. 4486.

[11] N.S. Manton. Phys. Rev. Lett. 1988. V. 60. P. 1916.

[12] V.D. Tsukanov. J. Phys. A. 1992. V. 25. P. 6099.

[13] R. Boesch, P. Stancioff, C. R. Willis. Phys. Rev. B. 1988. V. 38 . P. 6713.

[14] R. Boesch, C. R. Willis. Phys. Rev. B. 1990. V. 42. P. 2290.

[15] A. C. Newell, J. A. Whitehead. J. Fluid Mech. 1969. V. 38. P. 239

[16] T. Kavahara, M. Tanaka. Phys. Lett. A. 1983. V. 97. P. 311.

[17] Р. Раджсараман. Солитоны и инстантоны в квантовой теории. М.: Мир, 1985. 